\title{
UNIVERSITYOF BIRMINGHAM

\section{Live-imaging in the CNS: new insights on oligodendrocytes, myelination, and their responses to inflammation}

\author{
Rassul, Sayed; Neely, Robert; Fulton, Daniel
}

DOI:

10.1016/j.neuropharm.2015.09.011

License:

Creative Commons: Attribution-NonCommercial-NoDerivs (CC BY-NC-ND)

\section{Document Version}

Peer reviewed version

Citation for published version (Harvard):

Rassul, S, Neely, R \& Fulton, D 2015, 'Live-imaging in the CNS: new insights on oligodendrocytes, myelination, and their responses to inflammation', Neuropharmacology. https://doi.org/10.1016/j.neuropharm.2015.09.011

Link to publication on Research at Birmingham portal

Unless a licence is specified above, all rights (including copyright and moral rights) in this document are retained by the authors and/or the copyright holders. The express permission of the copyright holder must be obtained for any use of this material other than for purposes permitted by law.

-Users may freely distribute the URL that is used to identify this publication.

- Users may download and/or print one copy of the publication from the University of Birmingham research portal for the purpose of private study or non-commercial research.

- User may use extracts from the document in line with the concept of 'fair dealing' under the Copyright, Designs and Patents Act 1988 (?)

- Users may not further distribute the material nor use it for the purposes of commercial gain.

Where a licence is displayed above, please note the terms and conditions of the licence govern your use of this document.

When citing, please reference the published version.

Take down policy

While the University of Birmingham exercises care and attention in making items available there are rare occasions when an item has been uploaded in error or has been deemed to be commercially or otherwise sensitive.

If you believe that this is the case for this document, please contact UBIRA@lists.bham.ac.uk providing details and we will remove access to the work immediately and investigate.
} 
Live-Imaging in the CNS: New Insights on Oligodendrocytes, Myelination, and their Responses to Inflammation

\title{
Rassul Muhammed Sayed ${ }^{1}$, Robert K. Neely ${ }^{2}$, Daniel Fulton ${ }^{3}$
}

\author{
${ }^{1}$ Physical Sciences of Imaging in the Biomedical Sciences Training Programme, \\ University of Birmingham, Birmingham, UK. \\ ${ }^{2}$ School of Chemistry, University of Birmingham, Birmingham, UK. \\ ${ }^{3}$ Neurotrauma Research Group, Neurobiology Section, School of Clinical and \\ Experimental Medicine, College of Medical and Dental Sciences, University of \\ Birmingham, Birmingham, UK
}

Correspondence to: Daniel Fulton, Ph.D. Neurotrauma Research Group, Neurobiology Section, School of Clinical and Experimental Medicine, College of Medical and Dental Sciences, University of Birmingham, Edgbaston, Birmingham, B152TT. Email:

d.fulton@bham.ac.uk. Tel:+44-1214-148441.

\section{Highlights:}

1. Progress in live-imaging of oligodendrocyte-axon interactions and injury

2. Advantages and disadvantages of available platforms (in vitro, ex vivo, in vivo)

3. Applications of single molecule imaging in oligodendrocyte biology

4. Future opportunities, and practical applications, for live-imaging of oligodendrocyte and myelin injury responses and repair

This is the accepted version of the following article: Sayed, R.M., Neely, R.K., Fulton D. 2015. Live-Imaging in the CNS: New Insights on Oligodendrocytes, Myelination, and their Responses to Inflammation. Neuropharmacology. DOI: 10.1016/j.neuropharm.2015.09.011 


\begin{abstract}
The formation and repair of myelin involves alterations in the molecular and physical properties of oligodendrocytes, and highly coordinated interactions with their target axons. Characterising the nature and timing of these events at the molecular and cellular levels illuminates the fundamental events underlying myelin formation, and provides opportunities for the development of therapies to replace myelin lost through traumatic injury and inflammation. The dynamic nature of these events requires that live-imaging methods be used to capture this information accurately and completely. Developments in imaging technologies, and model systems suitable for their application to myelination, have advanced the study of myelin formation, injury and repair. Similarly, new techniques for single molecule imaging, and novel imaging probes, are providing opportunities to resolve the dynamics of myelin proteins during myelination. Here, we explore these developments in the context of myelin formation and injury, identify unmet needs within the field where progress can be advanced through live-imaging approaches, identify technical challenges that are limiting this progress, and highlight practical applications for these approaches that could lead to therapies for the protection of oligodendrocytes and myelin from injury, and restore myelin lost through injury and disease.
\end{abstract}

Keywords: Live-imaging, Single Molecule Imaging, Oligodendroycte, Myelination, Demyelination, Inflammation 


\section{Introduction}

CNS inflammation is the most common threat to myelin integrity, being involved in the pathology of multiple sclerosis (MS), a highly prevalent and incurable neurological disorder (Sospedra and Martin, 2005), and traumatic brain injury, which represents the most common form of morbidity in young adults worldwide (Werner and Engelhard, 2007). Injury to the myelin insulation on axons (demyelination) produces alterations in neuronal function that lead to neurological impairments compromising the health and wellbeing of patients (Compston and Coles, 2002). Consequently, inflammatory injury to myelin represents a key clinical challenge, and an area in which the development of novel regenerative therapies offer significant opportunities to improve the wellbeing of millions of individuals globally. Myelination involves coordinated molecular and physical interactions between oligodendrocytes (OL) and their target axons. A clearer definition of these events could expose information that may facilitate the development of novel therapies aiming to replace myelin damaged by inflammatory insults.

Until recently, technical challenges have restricted the analysis of these events to largely static modes of study such as immunofluorescence and electron microscopy (EM), which although capable of delivering detailed descriptions of OL-axon interactions, do not reveal their temporal dynamics as they unfold within living CNS structures. Advances in imaging technologies, including two-photon (2P) imaging, Single Molecule Imaging (SMI) approaches, and photoswitchable / convertible fluorescent proteins (FP), in combination with novel platforms for their implementation within ex vivo and in vivo CNS systems, are providing a new, more dynamic, viewpoint on the molecular and cellular dynamics that drive OL-axon interactions and myelination. Having brought new light to important questions of myelin formation, such as the temporal constraints governing the generation of new myelin internodes by differentiated OL, and the dynamics of individual myelin proteins as they self-associate within the developing myelin sheath, these novel molecular and cellular imaging techniques are now on the cusp of enabling similar advances to our understanding of OL and myelin injury. In this review, we explore the new knowledge provided by these imaging approaches, identify 
unmet needs where progress can be advanced with these methodologies, highlight technical challenges that are limiting this progress, and discuss practical applications that could improve our understanding of myelin injury, and provide novel data to advance the development of therapies for the restoration of myelin lost through inflammatory disease.

\section{Model systems for live-imaging OL dynamics, OL-Axon interactions, and myelination}

\subsection{In vitro primary cell systems}

Co-cultures of CNS-derived OL and neurons offer several advantages for live-imaging studies. First, purified CNS-derived OL precursor (OPC) and OL are widely used for live-imaging of migration, process dynamics and calcium signaling (Gudz et al., 2006; Paez et al., 2009, Xiao et al., 2013), thus a critical mass of knowledge exists supporting their use in further imaging studies assessing OL-axon interactions. Second, the timing of OPC seeding and differentiation can be controlled precisely (Watkins et al. 2008), facilitating observations of the initial stages of OPC-axon interactions and myelination. Third, adherent growth on coverslips, and a tolerance for submerged culture conditions, makes these cultures ideal for stable live-imaging over the extended periods of time required to capture OL processes growth and myelin segment formation. Fourth, OPCgenerating neurospheres, expressing FP, may be seeded onto neuronal cultures to generate fluorescently labeled OPC and OL without the requirement for maintaining costly transgenic colonies (Ioannidou et al., 2012). Despite these advantages, in vitro OLneuron systems represent an underexploited opportunity in the live imaging of OL-axon interactions and myelination. Neurosphere-derived OPC expressing membrane targeted GFP have been used to image the initial stages of OPC-axon contact (Ioannidou et al. 2012). In these time-lapse experiments, these interactions began with OPC processes spiraling around the target axons, before the development of membrane swellings that migrated along presumed axons, and occasionally fused, suggesting the deposition of proto-myelin materials (Figure 1). Imaging of cortical OPC-retinal ganglion cell cultures 
has provided more detail on the early stages of myelination by revealing that newly differentiated OL exhibit a brief time-window for the initiation of new myelin segments (6-12 hours), after which OL process dynamics are restricted to the remodeling of preexisting myelin segments (Watkins et al., 2008). Neuronal activity influences OPC development and myelination (Demerens et al., 1996; Fannon et al., 2015; Stevens et al., 2002; Wake et al., 2011), although the molecular events involved in these actions remain unclear. Live-imaging of myelinating OPC-dorsal root ganglion (DRG) co-cultures has helped to illuminate these events by revealing the influence of action potentials in directing the local translation and localisation of MBP (Wake et al., 2011). Here, the authors used an MBP fusion protein containing a photoconvertible FP that switches emissions from green to red wavelengths when stimulated with UV light. Using this tool, the authors showed that neuronal activity promoted the local synthesis of MBP, as revealed by imaging of newly produced red fluorescent MBP, and that this synthesis occurred locally within OPC processes. Further experiments were performed to determine the influence of axonal activity on the mobility of newly synthesized MBP by the creation of a second fusion protein, this time featuring a photoactivatable GFP, whose emission is dependent on UV light. Time-lapse studies revealed that MBP spots were highly localized within discrete locations in the process, and that blockade of electrical activity released the MBP enabling it to migrate freely. Together, these observations support a model in which action potentials stimulate the synthesis and localization of MBP at OPC process sites that could subsequently develop into myelin segments.

Despite the potential influences on cellular function arising from dissociation and subsequent culturing procedures, co-cultures have emerged as valuable models for both the study of myelination (Jarjour et al. 2012), and as useful platforms for live-imaging studies of OL-axon interactions and myelin formation. Consequently, we review their potential use in live-imaging studies of OL and myelin injury in section 2.4.1.

\subsection{Ex vivo CNS tissue systems}


Ex vivo systems preserve the cellular architecture of the CNS, and so offer a more faithful model of OL-axon interactions than in vitro systems established from isolated primary cells. Ex vivo preparations used to image OL process dynamics, axon interactions and myelination comprise acute (Haberlandt et al. 2011) and cultured brain slices (Haber et al., 2009; Harrer et al., 2009), and spinal cord explants (Ioannidou et al. 2012). Viable slice cultures of CNS tissue can be maintained in culture for a number of weeks (Haber et al., 2009; Simoni and Yu, 2006), and so provide ample opportunities to observe OL and axon dynamics at time-points remote enough from slice preparation to avoid the influence of slice-associated injuries. Acute slices maintained in well-oxygenated buffer remain viable for up to 10 hours (Fonseca et al., 2006), so can be used to monitor OL behaviours occurring on shorter time-scales, such as migration and OL process extension (Haberlandt et al., 2011). Slower events, such as myelin formation, injury and repair cannot be usefully studied over such short periods, so remain the preserve of slice cultures when ex vivo methods are sought. On the other hand, acute slices are readily generated from adult tissue, as opposed to slice cultures, which are generally not successful when obtained from adult tissue. Therefore, acute slices prepared from transgenic OL reporter mice provide an as yet unexplored opportunity for imaging of adult OPC and OL dynamics. NG2-eYFP reporter mice have been used to study OPC process dynamics in acute slices obtained from the developing hippocampus (Haberlandt et al., 2011). Here, time-lapse 2P confocal imaging reveals OPC processes extension and retraction events that occur on minute time-scales. Such exploratory behaviours match those observed for OPC in vivo (Section 2.3.1), but contrast with findings from hippocampal slice cultures, where OPC process remodeling is only apparent on hour time-scales (Haber et al., 2009). Interestingly, this slice culture study reveals that premyelinating OL show greater process remodeling than OPC, with marked process extension and retraction events occurring on the minute scale, with OL growth cones representing areas with the greatest activity. Haber et al. (2009) used a recombinant semliki forest virus (SFV) vector (type A7(74)) to deliver farnesylated GFP (GFPf) to OPC and OL in their slice cultures. GFPf delivered in this way labeled the fine processes of OPC and myelin segments on pro-myelinating and myelinating OL. Live-imaging of developing myelin segments revealed the extension and retraction of fine filopodia from 
these ensheathments, with new OL processes persisting for several hours. The function of these process extensions, and whether they are also apparent on more mature ensheathments awaits further investigation since similar observations are yet to be reported from other time-lapse studies. Varicosities are frequently observed along the processes of immature OL (Fulton et al., 2010). These swellings have been shown to contain enlarged mitochondria (Berger et al., 1991) and may therefore represent foci of enhanced metabolic activity, as may be expected at sites with ongoing membrane dynamics. Time-lapse imaging of hippocampal NG2-eYFP OPC (Haberlandt et al., 2011) revealed that these varicosities are motile, roaming over a number of micrometers within a few minutes. These motile swellings may be similar to the GFP filled spheres observed to travel along processes in cultured cerebellar slices isolated from a PLPeGFP reporter mouse (Sobottka et al., 2011). In this case the swellings were seen to travel from the soma out to processes, perhaps reflecting the supply of PLP to growing myelin segments. Further study is required to characterize the nature of these migratory swellings, and determine their association with sites of de novo process extension and myelin formation.

Transplantation of FP labeled OPC provides a useful strategy when developing ex vivo models for live-imaging. This approach revealed the initiation of OL-axon contact and myelination using OPC-generating neurospheres expressing either cytosolic GFP (GFPc) or membrane targeted GFPf (Ioannidou et al. 2012). Here, transgenic neurospheres were transplanted into the spinal cord of young shiverer mice, a popular donor for exogenous OPC due to the absence of MBP (Goldman et al. 2012). In addition, shiverer mice were crossed with a Thy1-CFP line to enable imaging of axons. Having confirmed their earlier in vitro findings (Section 2.1) that transplanted cells generate $\mathrm{MBP}^{+}$myelinating $\mathrm{OL}$ whose processes spiral around target axons, acute spinal cord explants were imaged to examine the steps leading from OL-axon contact to myelination. Imaging of GFPc revealed that extending OL processes initially establish axonal contact at multiple sites, before spreading laterally along the axon (Fig. 1A). Imaging of the GFPf tag proved more suitable for examining thickened nascent myelin segments, which exhibited bubble like membrane protrusions, whose migration and fusion may play a role in the early stages of myelin segment formation. This idea is supported by time-lapse observations from 
cerebellar slice cultures, where similarly dynamic membrane bubbles are apparent on the

processes of pro-myelinating and mature OL (D. Fulton, unpublished observation). These observations illustrate the advantage of time-lapse imaging in evaluating the models proposed to explain how axonal myelin ensheathment proceeds. The imaging data from Ioannidou et al. (2012) provide partial support for both the 'serpent' or 'coiled spring' model of myelin formation (Pedraza et al., 2009), where pre-myelinating OL processes initially spiral around axons before expanding laterally to form myelin segments, and the 'liquid croissant' model, developed from time-lapse data obtained from hippocampal slice cultures (Sobottka et al., 2011). In this scheme, a triangular shaped OL process wraps the axon, starting with the inner tongue, and producing an uneven surface with the appearance of regularly spaced spirals that spread the sheath bi-directionally from the initial point of contact. Importantly, it is the uneven spiral-like appearance of developing myelin that unifies the data obtained form these live-imaging studies. Further insights into this interesting subject have been obtained from in vivo imaging approaches (Snaidero et al., 2014). These developments are discussed in section 2.3.2.

\subsection{In vivo systems for imaging OL dynamics, axon interactions and myelination}

Challenges in achieving optical access to deep lying white matter tracts, where a large proportion of OL-axon interactions reside, has limited the progress of in vivo imaging studies in mammalian species. In contrast, progress in optically transparent, genetically tractable, zebrafish larvae have provided an alternative vertebrate system for the study of OL-axon interactions and myelination in the intact CNS. These subjects are reviewed in the following two sections.

\subsubsection{In vivo imaging of OPC migration and process dynamics in mammalian species}

Imaging OL-axon interactions within the intact mammalian CNS permits data to be obtained free from influences arising from long-term exposure to culture conditions, and provides the best opportunity to determine the impact of pathological conditions on the steps leading to myelin injury and repair within a clinically relevant context. These 
advantages come at a price, since in vivo imaging requires costly $2 \mathrm{P}$ optical setups to achieve useful excitation depths within the light-scattering environment of the CNS tissue (Misgeld and Kerschensteiner, 2006), complicated surgical procedures to obtain optical access to tissues, and equipment and expertise to ensure the welfare and viability of the anaesthetized animal. Imaging depth represents a significant challenge to in vivo OLaxon imaging in the brain, since a major proportion of these interactions arise in the deep lying white matter tracts. Complementary technologies, such as gradient index (GRIN) lenses, have extended the reach of $2 \mathrm{P}$ imaging to depths of $\sim 1 \mathrm{mM}$, enabling observations of white matter axonal bundles (Levene et al., 2004). However, despite these developments, they are yet to be used to achieve cellular resolution imaging of OL, or their interactions with target axons. Typically, 2P imaging is applied at depths up to $<500$ $\mu \mathrm{m}$ (Levene et al. 2004), which is sufficient to enable imaging of OPC and OL in more superficial grey matter regions (Hill and Grutzendler, 2014; Hughes et al., 2013). For example, an in vivo study of cortical OPC expressing membrane targeted GFP under control of the NG2 promoter, revealed these cells to possess dynamic growth cones and filopodia (Hughes et al., 2013) similar to those observed on OPC and immature OL in acute and cultured slices obtained from the developing CNS (Haber et al., 2009; Haberlandt et al., 2011). Adult OPC were also found to be highly motile, migrating into injury sites, and areas vacated by neighbouring OPC at rates of $\sim 2 \mu \mathrm{m} /$ day (Hughes et al., 2013). Migratory behaviours have been imaged for developmental OPC using cultured slices (Paez et al., 2009), but limitations on the viability of adult CNS slice cultures have until now limited the imaging of adult OPC motility. Consequently, 2P in vivo imaging has extended our understanding of OPC process and growth cone dynamics to adult OPC, and enabled new knowledge of adult OPC migration and injury responses, that were not possible with ex vivo techniques.

\subsubsection{In vivo imaging of OL dynamics, axon interaction and myelination in zebrafish}

Developments in the use of zebrafish have enabled significant advances in OL-axon that have been lacking in mammals. The embryonic and larval stages of these fish provide favourable targets for in vivo imaging due to their optical transparency, and the ease and 
low costs of their transgenesis into lines expressing cell-specific imaging probes. In addition, zebrafish generate myelin with a comparable composition and structure to that of mammals, but within an accelerated time-window ( $\sim 1$ week vs 4 weeks), thus representing an efficient and viable model for the study of myelination. These advantages, and the novel aspects of OL behavior revealed through zebrafish liveimaging have been reviewed in depth by Preston and Macklin (2015), therefore the following discussion is focused on findings from zebrafish that have advanced our understanding of OL process dynamics, axon interactions and myelin formation. Arguably the most influential study in this context is that of Kirby et al. (2006), who imaged spinal cord OPC providing the first real-time visualisation of CNS myelination initiation within an intact animal, and inspired a number of subsequent studies that have added significantly to our understanding of the OL-axon interactions and myelination (Czopka et al., 2013; Hines et al., 2015; Mensch et al., 2015; Snaidero et al., 2014). Kirby et al. (2006) showed that OPC labeled by the nkx2.2a:mGFP transgene, which drives expression of a membrane-targeted GFP, displayed rapid minute-scale process dynamics as they migrated towards axonal targets. Once migration ceased, process motility continued for some time before axon wrapping commenced, presumably indicating the initial stages of myelination. The transition from initial process contact to myelin segment formation has been resolved recently in the same line of transgenic fish (Czopka et al., 2013). Here, time-lapse data confirmed that the motile processes described by Kirby et al. (2006) develop into stable contacts that, over the course of a few hours, transition into thickened myelin segments. Building on their ability to image the formation of new myelin segments, the authors then used their time-lapse assay to investigate the temporal profile for myelin segment formation (Fig. 1B). By imaging the sequential formation of myelin segments from individual OL, and recording the time elapsing from OL differentiation, as indexed by the formation of the first myelin segment, to formation of the final myelin segment, it was determined that OL exhibit a surprisingly brief time-window for myelin segment formation of $\sim 5 \mathrm{~h}$ that mirrors previous observations from in vitro studies (Watkins et al., 2008). Interestingly, this parameter appears to be firmly set, since even manipulations of Fyn kinase activity that modulate the total number of myelin segments formed by OL, exhibit a period of 
effectiveness that is limited to this $5 \mathrm{~h}$ time-window (Czopka et al., 2013). The possibility that new internodal segments arise exclusively from newly differentiated OL may explain the failure of surviving OL to contribute to myelin repair (Keirstead and Blackemore, 1997), and highlights the mechanisms regulating this time-window as a potential target for pro-myelinating therapies. Without the use of time-lapse imaging it would not have been possible to observe these first glimpses of developing myelin segments, or derive the temporal limitations that influence their genesis.

Live-imaging studies in zebrafish have also advanced our understanding of the mechanisms by which the OL process wraps the target axon during myelination (Snaidero et al., 2014). Earlier ex vivo imaging studies (section 2.2) suggested that new myelin segments form from a central point in the future internode, before bi-directional lateral growth fills out the developing sheath (Sobottka et al., 2011). Here, the authors used in vivo imaging of developing internodes in nkx2.2a:mGFP larvae, combined with novel quantitative analyses linking fluorescent intensities to the number of myelin wraps, to confirm the centralised point of myelin formation, and provide real-time quantitative data to support the subsequent lateral extension and thickening of the internode. Despite supporting these aspects of the earlier ex vivo derived model, it should be noted that additional 3D analyses from serial block-face EM generated novel information that supported an outer-to-inner direction for the axial growth of the sheath that stands in marked contrast to the inner-to-outer scheme proposed by Sobottka et al. (2011).

New work in zebrafish larva has also provided fresh insights into the involvement of axonal activity in the formation of myelin. Neuronal activity influences myelination in the mammalian CNS (Demerens et al., 1996; Fannon et al., 2015; Gibson et al., 2014; Stevens et al., 2002 Wake et al., 2011), yet the specific stages of myelin formation that are influenced by activity remain unclear. Recent live-imaging studies of OL-axon interactions in zebrafish expressing novel tetanus toxin (TeNT) transgenes that silence axonal vesicular release have revealed an involvement of activity in a number of distinct stages of myelination. Specifically, disruption of activity-dependent OL-axon communication reduced the total number and length of myelin sheaths formed by 
individual OL (Mensch et al., 2015; Hines et al., 2015), altered the selection of axonal targets by OL, and compromised the maintenance of established sheaths (Hines et al., 2015).

Imaging of fixed samples has provided indirect evidence that neuronal activity enhances the extension and branching of OPC processes in cerebellar slice cultures (Fannon et al., 2015), but these observations are yet to be confirmed in living tissue. The TeNT transgenic fish described above provide an excellent opportunity to examine this question. Earlier studies using the sox10:RFP reporter line (Kirby et al., 2006) demonstrated that OPC exhibit highly motile processes during the time-frame spanning OPC specification to the initiation of axon wrapping (approximately 36 to 72 hours post fertilization (hpf)). The study by Hines et al. (2015) did not examine OPC process motility over these time-points (Hines J \& Appel B, personal communication), therefore live-imaging of process dynamics in a TeNT-sox10:RFP transgenic during this timewindow may reveal new information on the role neuronal activity plays in guiding the extension and retraction of OPC processes as they search their environment for suitable axonal targets.

Although this larvae based system has limited potential as a model for demyelination / remyelination in the adult CNS, the progress it has enabled in illuminating the physical and temporal dynamics of myelination place it in an excellent position to contribute to future studies examining the response of the developing OL / myelin system to injury.

\subsection{Live-imaging OL myelin injury responses and repair}

The experimental systems described in the preceding sections, and the results in OL-axon interactions and myelination they have enabled, provide the basis for investigations into the response of the axon-OL unit to inflammatory and traumatic injury. This area deserves increased attention since it offers the potential to further our understanding of the pathological events underlying the acute stages of demyelination, and offers exciting opportunities for the development of dynamic assays suitable for the screening of 
protective and regenerative therapies. Despite the clear benefits of live-imaging within an injury context, the literature consists of just a few papers, which have mainly provided qualitative levels of analysis. The following sections review work performed with in vitro cell cultures (section 2.4.1), ex vivo slice cultures (section 2.4.2) and in vivo models (section 2.4.3).

\subsubsection{In vitro cell culture imaging of OL and myelin injury}

OL-neuron co-cultures largely lack astrocyte, microglial and immune cell interactions, and so do not closely model in vivo myelin injury and repair. Despite this drawback, a simple model for autoimmune mediated OL and myelin injury suggests that they still hold value for future studies examining OL-axon interactions within the injury context (Pang et al. 2012). In this model, co-applications of MOG antibody and complement produced a robust demyelination in co-cultures of CNS-derived primary OPC and neurons. Additionally, the commonly used membrane-solubilizing agent lysolecithin can also be used to demyelinate these cultures (Pang et al. 2012). Complex cultures containing multiple neural cell types dissociated from the CNS can also provide a myelinating culture suitable for the study of myelin injury and repair (Boomkamp et al. 2012). Importantly, these systems retain microglia and astrocytes from the source tissue, and can be supplemented with additional astrocytes, providing a culture that more closely resembles the cellular environment within the intact CNS. Cultures of this type can be used to study myelin injury and repair. For example, Boomkamp et al. (2012) used embryonic rat spinal cord derived cells to develop a model of spinal cord injury capable of exhibiting demyelination and myelin repair following axonal transection. This model was shown to provide a useful system for screening pharmacological promoters of regeneration, with a combined treatment of Rho and ROCK inhibitors proving effective in driving axonal and myelin repair respectively.

Live-imaging studies are yet to be performed using these in vitro injury models. However, the widespread use of co-cultures for studies of myelination (Jarjour et al. 2012), and the extensive body of expertise and techniques associated with their use in 
imaging studies, suggests that in vitro systems have the potential to provide a useful platform for the imaging of OL and myelin injury that can complement more complex ex vivo and in vivo systems.

\subsubsection{Live-imaging OL and myelin injury in ex vivo slice cultures}

Two studies have used cerebellar slice cultures derived from PLPeGFP reporter mice to investigate the dynamics of myelin injury in the living CNS. The first example used an $e x$ vivo model of immune mediated demyelination to obtain the first documented live visualization of myelin injury and repair (Harrer et al., 2009). Here, PLPeGFP cerebellar slice cultures were injured with a MOG antibody / complement treatment. Imaging of propidium iodide labeled slices indicated that this treatment triggered widespread OL death, leading to demyelination, but sparing axons. This demyelination could be tracked using time-lapse imaging of the PLPeGFP reporter (Fig. 1C), enabling the spatiotemporal kinetics of demyelination to be visualised. Myelin injury began $\sim 2$ hours after the onset of the injury, appearing first at thin $\mathrm{GFP}^{+}$fibers located at tips of folia, before proceeding centrally towards thicker myelin profiles. Demyelination of thin peripheral processes was complete by around 16 hours, while denser areas of myelination proved more resistant, requiring longer treatments, with pronounced demyelination appearing after $>24$ hours. Following withdrawal of the injury treatment, slice cultures exhibited a robust remyelination response, which could also be monitored by time-lapse analysis. Remarkably, recovery could be observed $\sim 5$ hours after withdrawal of the injury treatment, appearing first with the growth of $\mathrm{GFP}^{+}$processes in regions containing $\mathrm{GFP}^{+}$ soma, which subsequently develops into a new array of myelinating fibers. That repair begins near residual OL suggests that newly forming processes and myelin are formed by OL that survive the injury, a situation that contrasts with in vivo work employing a similar injury treatment, when surviving OL in the spinal cord failed to regenerate compact myelin despite producing processes that engaged and wrapped axons. (Keirstead and Blackemore, 1997). Immunofluorescence for MBP and neurofilament indicates that regenerating $\mathrm{GFP}^{+}$processes in these slices do indeed myelinate target axons, raising 
interesting questions regarding the potential for surviving OL to contribute to myelin repair in different model systems and CNS regions.

Further insights into immune-mediated myelin injury have been generated from slice cultures isolated from PLPeGFP mice expressing the $\mathrm{CD} 8^{+} \mathrm{T}$ cognate antigen (ovalbumin peptide, OVA) within OL (PLPeGFP:OVA) (Sobottka et al., 2009). Here the authors developed a novel immune-mediated demyelinating model involving delivery of activated $\mathrm{CD} 8^{+} \mathrm{T}$ cells to PLPeGFP:OVA cerebellar slices pre-incubated in interleukin gamma ( $\left(\mathrm{FN}_{\Upsilon}\right)$, to activate MHC class I molecules on myelin. As well as producing extensive injury to myelin, the treatment also injured axons. The specific expression of OVA within OL, and the absence of MHC class I molecules on axons after IFN $\mathrm{N}_{\Upsilon}$ treatment suggested that this axonal injury was secondary to CD8-directed injury to myelin. In addition to these novel findings on CD8-mediated axonal loss, time-lapse imaging of DiD tagged CD8 cells, and PLPeGFP signals, revealed several novel aspects of CD8 cell dynamics within CNS tissue, including scanning behaviours (circular migration paths) that were absent in slices not exposed to IFN $\mathrm{N}_{\Upsilon}$, and associations with $\mathrm{OL}$ and myelin, that were frequently associated with demyelination.

Despite their purely qualitative nature, both studies from PLPeGFP slice cultures provide important advances by delivering the first glimpses of CD8 cell dynamics and myelin injury and repair in the living CNS, and providing valuable systems suitable for the screening of pro-regenerative treatments within the context of inflammation.

\subsubsection{In vivo imaging of OL injury and regeneration}

To date, there are no studies providing direct visualization of demyelination in an intact CNS. However, two recent imaging studies offer distinct views on the response of OL to CNS injury. The first uses Xenopus larvae, that offer similar optical and genetic advantages to zebrafish, to perform an in vivo investigation of OL injury and regeneration (Kaya et al., 2012). This work developed an MBP reporter line expressing eGFP fused to nitroreductase (MBP-eGFP-NTR), an enzyme that generates cytotoxins from the conversion of the harmless pro-drug metronidazole (MTZ). After initial studies 
confirming expression of the transgene within myelinating OL, the authors performed in vivo time-lapse MP imaging to detect the response of OL to MTZ induced injury. Imaging revealed a reduction in optic nerve $\mathrm{GFP}^{+} \mathrm{OL}$ after a 3-day treatment with $\mathrm{MTZ}$, with OL death being subsequently confirmed to arise through apoptosis. Further analysis by luxol blue staining, and EM, revealed that MTZ directed OL death was associated with demyelination that spared axons. Following withdrawal of MTZ, larvae exhibited spontaneous regeneration of $\mathrm{GFP}^{+} \mathrm{OL}$ that was captured by further time-lapse imaging. Subsequent EM analysis showed that this OL regeneration was associated with remyelination, although evidence connecting newly formed $\mathrm{GFP}^{+} \mathrm{OL}$ to myelin internodes was not sought. Importantly, the regenerative response was enhanced by retinoic acid, a known promoter of myelin formation and repair, providing proof of concept for the use of this system to screen potential regenerative enhancers. Added to this, demyelination and remyelination in this model is rapid, with each occurring within 3 days, thus overall, the MTZ model has good potential as a screening system for evaluating the repair kinetics of protective and regenerative therapies. While this model may provide a useful tool for assessing OL regeneration in the intact CNS, the eGFPNTR fusion strategy is not suitable for the visualization of myelin degeneration and repair as the cytosolic eGFP used is excluded from compacted myelin. Presumably a membrane targeted GFP fusion, capable of labeling mature myelin (e.g. Kirby et al., 2006), has not been used because membrane localization may impair the function of NTR. Future developments could use a strategy that permits the fusion partners to assume separate cellular distributions, for example a cleavable peptide linker (Provost et al., 2007), since this approach would enable the use of a membrane targeted GFP capable of probing compacted myelin. These developments would complement and extend the opportunities for Xenopus myelin regeneration studies that have already been demonstrated by Kaya et al. (2012).

The second in vivo imaging study to describe OL responses to CNS injury marks a departure from the other work reviewed since it utilizes imaging of bioluminescence rather than FP, and because the signals that are sampled represent the summed output from large areas of CNS tissue rather than discrete cells (Locatelli et al., 2015). Here, $\beta$ - 
actin expression was tracked specifically in mature OL by crossing a MOG-Cre line with mice expressing a Cre-inducible $\beta$-actin-luciferase construct. Luciferase signals from this double transgenic were then used as an indirect reporter of actin cytoskeleton dynamics in mature OL. Consistent with this strategy, in vivo luciferase activity from the spinal cord and brain increased steadily during postnatal development before reaching a steadystate in the adult, consistent with the generation of mature OL and myelin during this period. Having validated their in vivo assay for $\beta$-actin activity, the authors went on to examine how inflammatory CNS disease influences OL cytoskeletal dynamics by imaging $\beta$-actin activity in mice subjected to MOG peptide-induced experimental autoimmune encephalomyelitis (EAE). Mice exhibiting clinical disease showed a biphasic $\beta$-actin expression profile, with a sharp increase in expression that coincided with disease onset, before a decline to near pre-immunization levels. This profile suggests that at the outset of the disease, OL respond to inflammatory injury with an active rearrangement of the cytoskeleton, after which there is progressive loss of OL $\beta$-actin expression that is time-locked to the decline in clinical symptoms associated with the ameliorating remitting phase. In support of this interpretation, luciferase activity was not altered in EAE animals that did not exhibit clinical disease. The novel imaging approach used in this study enabled new insights into the response of OL during inflammatory demyelinating insults, indicating that OL are capable of mounting an actin-dependent injury response that could impart a degree of protection, and which may be exploited in protective therapies. The imaging methods lacked cellular resolution, so the study was not able to reveal whether and how these changes in $\beta$-actin expression were translated into changes in OL morphology, and myelin injury. Investigation of these questions at the cellular level will require new developments in the ability to achieve OL-myelin imaging within the rodent spinal cord and deep lying white matter tracts that are affected by MOG EAE (section 2.3.1). Therefore, ex vivo inflammatory demyelination models, in which it is possible to resolve OL and myelin injury dynamics, continue to offer an attractive alternative to in vivo studies (Harrer et al., 2009; Sobottka et al., 2009, section 2.4.2)

\subsection{Single molecule imaging (SMI) of myelin protein behaviour and function}




\subsubsection{Single molecule imaging}

SMI methods allow specific subsets of molecules to be studied one at a time. This enables the identification and analysis of discrete molecular behaviours that may be lost with traditional 'ensemble' based imaging approaches in which the sample is examined as a whole rather, than a collection of nano environments (Lord et al 2010). This is critical, particularly in biological systems, where a small percentage of a given population of molecules, such as an enzyme, may be active or responsible for the behaviour of interest. Underpinning every SMI approach is the requirement that individual molecules are either spatially- or temporally-resolved. This allows subpopulations of molecules to be identified, time-dependent molecular events to be explored, and nano environments within the system to be observed. These features of SMI are critical to improving our understanding of cell biology since events that are uncommon, or that exhibit tight temporal characteristics, could be essential for determining the function, or initiation, of a given cellular process (reviewed by Coelho et al., 2013). For these reasons single molecule strategies have been utilised to observe a spectrum of biological molecules ranging from single DNA strands to viruses (Lord et at 2010). In addition to elaborating the nano environment within a system, SMI allows particles to be tracked, providing information on their behaviour within the system, and their association to the time-frame. SMI also enables quantitative measures of the molecule in question because each molecule is attached to a probe, allowing parameters such as fluorescent intensity to be directly associated with molecular concentration.

A number of different fluorescence based probes are available for SMI including those based on quantum dots, synthetically prepared fluorophores, and genetically encoded FP. Techniques involving FP are the most commonly used since they offer a number of advantages over the other probes. Chief among these are their low toxicity, their ease of conjugation to proteins of interest, and their availability across a wide spectral range. Longer wavelength FP are more desirable, as they provide better contrast against background signals, which is a particular challenge for SMI, since the signal intensities 
involved are considerably lower than those in ensemble imaging. This problem is compensated through the use of novel photoswitchable and photoconvertible FP. Photoswitchable proteins undergo a reversible conformational change, allowing subsets of the FP to be switched between "on" and an "off" states of fluorescence, while photoconvertible proteins can be stimulated to undergo changes in their emission wavelength, enabling the isolation of a sub-population of FP in the sample.

These properties are highly useful in SMI because they enable individual fluorophores to be isolated and tracked in a localized region. This approach was demonstrated in the work of Aggarwal et al. (2013), where a laser was used to switch the photoconvertible fluorescent protein, Dendra2, in a central location within the sample from green to red emission. In this way a subset of molecules, localised to a defined area, could be investigated without the need to use multiple imaging probes or targets.

\subsubsection{Application of SMI in OL}

To date there are only a handful of notable papers which utilise fluorescent single molecule techniques. Here, we discuss those studies that have employed SMI techniques to derive influential findings on myelin protein and OL biology. The first pioneered the observation of intracellular RNA granule transport, which was performed on OL using SMI to view MBP mRNA transport (Ainger et al 1993). Here, fluorescein was fused to the 3' end of MBP mRNA, allowing individual MBP mRNA to be temporally tracked. Following injection, the tagged RNA construct was followed in primary OL by confocal microscopy. Time-lapse imaging demonstrated that MBP mRNA granules were produced in the perikaryon of myelinating OL, before being deposited into the peripheral margins of the myelin sheets demonstrating the effectiveness of SMI in live molecular tracking. Although spatially the individual molecules were not effectively resolved, this is still an example of SMI as the method allowed effective temporal resolution of the molecules. From these experiments the mRNA granules were found to be aligned in tracks within the OL cytoplasm and processes, suggesting they were transported using the cytoskeletal matrix. This theory was confirmed using antibody staining which showed MBP granules associated with the cytoskeleton. This could not have been found without the support of 
single molecule tracking, as immunostaining can only provide static images of the cell, making analysis of granule movements impossible. As one of the earliest papers utilising SMI techniques, it provides a vast amount of important information on mRNA vesicle dynamics, and the transport and localisation of MBP mRNA in OL.

An interesting example of the use of SMI to quantify a myelin protein, and investigate its regulation through molecular interactions is shown by Giampetruzzi et al. (2013). Here, the authors examined the influence of the fragile $\mathrm{X}$ mental retardation protein (FMRP) on MBP translation, because the absence of FMRP is associated with deficits in CNS myelination. To study this observation they used a chimeric mRNA construct encoding an MBP-venus fusion protein. A cy5 UTP label on this mRNA construct enabled its localisation and tracking within mRNA granules, while the venus FP served as a translation reporter for MBP. To determine how FMRP influenced MBP translation, venus fluorescence was measured in granules exhibiting different levels of endogenous FMRP expression. Importantly, venus expression was measured at the single molecule level by fluorescent correlation spectroscopy (FCS), which utilises the Brownian motion of single fluorophore labelled molecules to derive a concentration of the molecule. This method showed that FMRP only inhibited MBP at non-physiologically relevant levels $(>168 \mathrm{nM})$, leading to the conclusion that FMRP did not represent an important regulator of MBP translation in OL. The use of photobleaching with single molecule labelling allowed reliable photon counts to be achieved using FCS. Without the SMI strategy the signal from each granule would be too large to count, and the result would not have accurately quantified MBP translation.

Arguably the most notable paper describing SMI within OL reports recent studies into the self-association of MBP proteins within the inner leaflet of the myelin sheath (Aggarwal et al 2013). Here, a chimeric MBP construct, featuring an integral membrane protein domain connected to both GFP and MBP (GFP-TM-MBP), was employed as a reporter for tracking MBP diffusional mobility within primary OL. After initially confirming the functionality of this construct in OL derived from shiverer MBP mutants, the authors used FRAP (fluorescence recovery after photobleaching) assays to demonstrate that MBP 
reduces the diffusion of the GFP-TM reporter, since FRAP was markedly reduced when GFP was present in the construct (Fig. 2D, 2E). These observations were extended by fluorescence decay after photoconversion experiments involving a fusion construct containing dendra2 in place of GFP (GFP-TM-dendra2) (Fig. 2A-C). Dendra2 was chosen because photoconverted signals can be easily restricted to a portion of the sample, enabling individual particles within the sample to be tracked. Comparisons of time-lapse data obtained from reporter constructs containing and lacking MBP confirmed that the myelin protein reduced the diffusional properties of the reporter protein, potentially due to self-association of MBP molecules arising from hydrophobic interactions. This hypothesis was examined using a combination of FRET (Forster Resonance Energy Transfer) based SMI techniques, and MBP mutants carrying amino acid substitutions that replaced phenylalanine residues, needed for hydrophobic interactions, with non hydrophobic residues. In this version of the FRET assay protein-protein interactions are assessed by monitoring the fluorescence intensity of a donor FP following photobleaching of the acceptor FP. If interactions exist then acceptor bleaching should provoke a rise in intensity in donor FP intensity. Here, bleaching of the acceptor MBP-FP rapidly induced increases in the intensity of the donor MBP-FP, indicating close interactions between these MBP molecules. However, when the assay was performed using mutant MBP, the FRET signals were much weaker indicating that hydrophobic interactions between MBP molecules were important for their interactions and selfassembly. Importantly, the authors demonstrated the function of these interactions by examining the ability of MBP to extrude CNPase from the myelin sheet. Mutant MBP lacking the ability to self assemble via hydrophobic interactions proved ineffective in extruding CNPase. Thus, a combination of SMI based time-lapse imaging approaches and photoswitchable FPs revealed the behaviour of MBP molecules on the nano-scale, and illuminated molecular interactions that are necessary for MBP function.

\subsubsection{Potential for Single molecule imaging of OL injury and disease}

Although to date SMI methods have not been used in studies of OL and myelin injury, these techniques have many potential uses in this context. First, SMI methods allow 
higher resolutions on systems previously considered as lower resolution, and yet are not encumbered with the disadvantages typically associated with conventional highresolution systems, such as the electron microscope, where sample conditions must be in non-native, non-ambient conditions, and where dynamic time-lapse sampling is impossible. These advantages have clear benefits when seeking to investigate the molecular dynamics associated with injury in inflammatory and traumatic myelin injury models, where real-time data has so far been limited to cellular level imaging resolutions and qualitative analyses (Harrer et al., 2009, Kaya et al., 2012). The increased spatiotemporal information provided from time-lapse SMI could build upon the developmental aspects of myelination already advanced through conventional liveimaging studies (Czopka et al 2013; Hines et al., 2015; Kirby et al., 2006; Mensch et al., 2015; Snaidero et al., 2014), and allow new insight into the nature and timing of molecular changes occurring in experimental models of inflammatory and traumatic myelin injury.

Second, the use of SMI-based molecular tracking, in association with photo-switchable FP, could deliver significant advances in our understanding of myelin injury and repair. For example, SMI-tracking of individual myelin proteins, and their regulatory pathways, could facilitate investigations into the molecular dynamics underlying myelin degeneration and remyelination. Such developments could also be used to track the interactions of myelin proteins with signalling and structural molecules within the target axon, as shown in the work of Zonta et al. (2008), where it was demonstrated that Neurofascins have a distinct role in the assembly of the nodes of Ranvier.

Third, SMI provides the opportunity to determine quantitative descriptions of disease states, and treatment efficacy, by measuring molecular concentrations, diffusion coefficients, and reaction rates. Such developments could provide valuable additions to the methods traditionally employed in clinical diagnostic work, and disease epidemiology. 
Although available SMI methods undoubtedly offer advantages for the study of myelin injury and repair, there is still potential to improve on these techniques. SMI could benefit from increased imaging speeds to improve tracking studies, and to enhance the resolution of fine molecular details involved in dynamic events such as OL process motility. While SMI can be performed on systems as simple as epifluorescent microscopes if slow, well labeled, molecules with low concentrations are used, advances in SMI techniques on non-widefield imaging platforms, such as the light sheet microscope, would allow imaging over extended periods of time, potentially allowing observations of events such as myelination to be studied at finer resolutions. In association with single molecule studies, further use of photo activatable/inactivatable molecules would enhance both the field of SMI, and studies into OL and myelin, as they could allow multiple events to be probed more intricately in a spatiotemporal manner, and enable better-localised investigations on the effects of molecules. Another key area for improvement is the development of higher throughput techniques for SMI. Current technologies for SMI do not allow multiple molecules to be followed simultaneously, so advances in this area would significantly increase the capacity of SMI studies. Finally, a logical ambition capable of advancing our understanding of OL and myelin injury is the ability to perform in vivo SMI. A reasonable intermediate step for this could be provided by ex vivo systems, such as cultured slices containing deeper lying white matter tracts, which retain the circuits and neural cellular interactions of the intact CNS, but where access to imaging is relatively uncomplicated. Encouragingly, in vivo SMI has already been achieved, albeit from non-CNS tissues (Ritter et al. 2010), thus hopes of further SMI developments in the CNS appear reasonable.

\section{Conclusion}

Advances in imaging technologies, and their implementation within CNS models supporting OL development and myelination, most notably transgenic zebrafish, have enabled the visualisation of OL process dynamics and axon interactions within living CNS tissues (Figure 3). A number of studies have exploited these opportunities to observe the spatio-temporal dynamics of myelination, leading to new knowledge on the 
nature and timing of events that arise during myelin formation. Parallel developments in SMI techniques, and the novel FP that support them, are enabling discoveries on the behaviour and function of individual myelin proteins during myelination (Figure 3). However, SMI represents an under exploited area of work in the context of OL biology, and the potential for novel discoveries is considerable. Continued improvements in SMI, including increased imaging speed, and higher-throughput methods enabling the tracking of multiple FPs, will certainly facilitate these opportunities. Less progress has been made in imaging the response of OL and myelin to CNS injury. In this context slice culture studies have lead the way, while in vivo imaging in mammalian CNS white matter tracts remains challenging. Although live-imaging of OL and myelin in superficial grey matter regions is likely to be achieved soon, the goal of visualizing the deeper structures remains an important ambition if the field is to acquire in vivo imaging platforms suitable for monitoring the response of OL and myelin during experimental inflammatory insults such as EAE, which predominantly attack myelin in the white matter regions of spinal cord and brainstem. An alternative to this approach is the development of inflammatory models of demyelination in zebrafish or Xenopus larvae, for which the technologies for in vivo myelin imaging already exist. However, the modeling of an adult CNS condition such as inflammatory demyelination, within developing CNS that largely lack adaptive immune systems, raises considerable limitations that should be considered carefully. Notwithstanding these caveats, the potential to study inflammatory OL-axon injury in the intact CNS would still provide exciting opportunities for discovery. In conclusion, success in either the mammalian models, or lower vertebrates such as zebrafish and Xenopus larva, would lay the foundations for dynamic assays capable of screening therapies to protect OL and myelin from inflammatory injury, and restore myelin lost through injury and disease.

\section{Acknowledgements}

We thank Professor Martin Berry for comments on the manuscript. SMR gratefully acknowledges financial support from the EPSRC through a studentship from the PSIBS Doctoral Training Centre (EP/F50053X/1). RKN was supported by funds from the 
Higher Education Funding Council for England (HEFCE). DF dedicates this paper to the memory of Nick Talbot, a good friend, talented musician, and exceptional thinker.

\section{References}

Aggarwal, S., Snaidero, N., Pahler, G., Frey, S., Sanchez, P., Zweckstetter, M., Janshoff, A., Schneider, A., Weil, M. T., Schaap, I. A., Gorlich, D., Simons, M., 2013. Myelin membrane assembly is driven by a phase transition of myelin basic proteins into a cohesive protein meshwork. PLoS Biol 11, e1001577.

Ainger, K., Avossa, D., Morgan, F., Hill, S. J., Barry, C., Barbarese, E., Carson, J. H., 1993. Transport and localization of exogenous myelin basic protein mRNA microinjected into oligodendrocytes. J Cell Biol 123, 431-441.

Berger, T., Schnitzer, J., Kettenmann, H., 1991. Developmental changes in the membrane current pattern, $\mathrm{K}+$ buffer capacity, and morphology of glial cells in the corpus callosum slice. J Neurosci 11, 3008-3024.

Boomkamp, S. D., Riehle, M. O., Wood, J., Olson, M. F., Barnett, S. C., 2012. The development of a rat in vitro model of spinal cord injury demonstrating the additive effects of Rho and ROCK inhibitors on neurite outgrowth and myelination. Glia 60, 441456.

Coelho, M., Maghelli, N., Tolic-Norrelykke, I. M., 2013. Single-molecule imaging in vivo: the dancing building blocks of the cell. Integr Biol (Camb) 5, 748-758.

Compston, A., Coles, A., 2002. Multiple sclerosis. Lancet 359, 1221-1231.

Czopka, T., Ffrench-Constant, C., Lyons, D. A., 2013. Individual oligodendrocytes have only a few hours in which to generate new myelin sheaths in vivo. Dev Cell 25, 599-609. 
De Simoni, A., Yu, L. M., 2006. Preparation of organotypic hippocampal slice cultures: interface method. Nat Protoc 1, 1439-1445.

Demerens, C., Stankoff, B., Logak, M., Anglade, P., Allinquant, B., Couraud, F., Zalc, B., Lubetzki, C., 1996. Induction of myelination in the central nervous system by electrical activity. Proc Natl Acad Sci U S A 93, 9887-9892.

Fannon, J., Tarmier, W., Fulton, D., 2015. Neuronal activity and AMPA-type glutamate receptor activation regulates the morphological development of oligodendrocyte precursor cells. Glia.

Fonseca, R., Nagerl, U. V., Bonhoeffer, T., 2006. Neuronal activity determines the protein synthesis dependence of long-term potentiation. Nat Neurosci 9, 478-480.

Fulton, D., Paez, P. M., Fisher, R., Handley, V., Colwell, C. S., Campagnoni, A. T., 2010. Regulation of L-type $\mathrm{Ca}++$ currents and process mophology in white matter oligodendrocyte precursor cells by golli-myelin proteins. Glia 58, 1292-1303.

Giampetruzzi, A., Carson, J. H., Barbarese, E., 2013. FMRP and myelin protein expression in oligodendrocytes. Mol Cell Neurosci 56, 333-341.

Gibson, E. M., Purger, D., Mount, C. W., Goldstein, A. K., Lin, G. L., Wood, L. S., Inema, I., Miller, S. E., Bieri, G., Zuchero, J. B., Barres, B. A., Woo, P. J., Vogel, H., Monje, M., 2014. Neuronal activity promotes oligodendrogenesis and adaptive myelination in the mammalian brain. Science (New York, N Y ) 344, 1252304.

Goldman, S. A., Nedergaard, M., Windrem, M. S., 2012. Glial progenitor cell-based treatment and modeling of neurological disease. Science 338, 491-495. 
Gudz, T. I., Komuro, H., Macklin, W. B., 2006. Glutamate stimulates oligodendrocyte progenitor migration mediated via an alphav integrin/myelin proteolipid protein complex. J Neurosci 26, 2458-2466.

Haber, M., Vautrin, S., Fry, E. J., Murai, K. K., 2009. Subtype-specific oligodendrocyte dynamics in organotypic culture. Glia 57, 1000-1013.

Haberlandt, C., Derouiche, A., Wyczynski, A., Haseleu, J., Pohle, J., Karram, K., Trotter, J., Seifert, G., Frotscher, M., Steinhauser, C., Jabs, R., 2011. Grey matter NG2 cells display multiple $\mathrm{Ca} 2+$-signaling pathways and highly motile processes. PloS one 6 , e17575.

Harrer, M. D., von Budingen, H. C., Stoppini, L., Alliod, C., Pouly, S., Fischer, K., Goebels, N., 2009. Live imaging of remyelination after antibody-mediated demyelination in an ex-vivo model for immune mediated CNS damage. Exp Neurol 216, 431-438.

Hill, R. A., Grutzendler, J., 2014. In vivo imaging of oligodendrocytes with sulforhodamine 101. Nat Methods 11, 1081-1082.

Hines, J. H., Ravanelli, A. M., Schwindt, R., Scott, E. K., Appel, B., 2015. Neuronal activity biases axon selection for myelination in vivo. Nat Neurosci 18, 683-689.

Hughes, E. G., Kang, S. H., Fukaya, M., Bergles, D. E., 2013. Oligodendrocyte progenitors balance growth with self-repulsion to achieve homeostasis in the adult brain. Nat Neurosci 16, 668-676.

Ioannidou, K., Anderson, K. I., Strachan, D., Edgar, J. M., Barnett, S. C., 2012. Timelapse imaging of the dynamics of CNS glial-axonal interactions in vitro and ex vivo. PloS one 7, e30775. 
Jarjour, A. A., Zhang, H., Bauer, N., Ffrench-Constant, C., Williams, A., 2012. In vitro modeling of central nervous system myelination and remyelination. Glia 60, 1-12.

Kaya, F., Mannioui, A., Chesneau, A., Sekizar, S., Maillard, E., Ballagny, C., HouelRenault, L., Dupasquier, D., Bronchain, O., Holtzmann, I., Desmazieres, A., Thomas, J. L., Demeneix, B. A., Brophy, P. J., Zalc, B., Mazabraud, A., 2012. Live imaging of targeted cell ablation in Xenopus: a new model to study demyelination and repair. $\mathrm{J}$ Neurosci 32, 12885-12895.

Keirstead, H. S., Blakemore, W. F., 1997. Identification of post-mitotic oligodendrocytes incapable of remyelination within the demyelinated adult spinal cord. J Neuropathol Exp Neurol 56, 1191-1201.

Kirby, B. B., Takada, N., Latimer, A. J., Shin, J., Carney, T. J., Kelsh, R. N., Appel, B., 2006. In vivo time-lapse imaging shows dynamic oligodendrocyte progenitor behavior during zebrafish development. Nat Neurosci 9, 1506-1511.

Levene, M. J., Dombeck, D. A., Kasischke, K. A., Molloy, R. P., Webb, W. W., 2004. In vivo multiphoton microscopy of deep brain tissue. J Neurophysiol 91, 1908-1912.

Locatelli, G., Baggiolini, A., Schreiner, B., Palle, P., Wasiman, A., Becher, B., Buch, T., 2015. Mature oligodendrocytes actively increase in vivo cytoskeletal plasticity following CNS damage. Journal of Neuroinflammation 12. DOI:10.1186/s12974-12015-1027112972.

Lord, S. J., Lee, H. L., Moerner, W. E., 2010. Single-molecule spectroscopy and imaging of biomolecules in living cells. Anal Chem 82, 2192-2203.

Mensch, S., Baraban, M., Almeida, R., Czopka, T., Ausborn, J., El Manira, A., Lyons, D. A., 2015. Synaptic vesicle release regulates myelin sheath number of individual oligodendrocytes in vivo. Nat Neurosci 18, 628-630. 
Misgeld, T., Kerschensteiner, M., 2006. In vivo imaging of the diseased nervous system. Nat Rev Neurosci 7, 449-463.

Paez, P. M., Fulton, D. J., Spreuer, V., Handley, V., Campagnoni, C., Macklin, W. B., Colwell, C. S., Campagnoni, A. T., 2009. Golli myelin basic proteins regulate oligodendroglial progenitor cell migration through voltage-operated $\mathrm{Ca}++$ influx. $\mathrm{J}$ Neurosci 20, 6663-6676.

Pang, Y., Zheng, B., Kimberly, S. L., Cai, Z., Rhodes, P. G., Lin, R. C., 2012. Neuronoligodendrocyte myelination co-culture derived from embryonic rat spinal cord and cerebral cortex. Brain Behav 2, 53-67.

Pedraza, L., Huang, J. K., Colman, D., 2009. Disposition of axonal caspr with respect to glial cell membranes: Implications for the process of myelination. J Neurosci Res 87, 3480-3491.

Preston, M. A., Macklin, W. B., 2015. Zebrafish as a model to investigate CNS myelination. Glia 63, 177-193.

Provost, E., Rhee, J., Leach, S. D., 2007. Viral 2A peptides allow expression of multiple proteins from a single ORF in transgenic zebrafish embryos. Genesis 45, 625-629.

Ritter, J. G., Veith, R., Veenendaal, A., Siebrasse, J. P., Kubitscheck, U., 2010. Light sheet microscopy for single molecule tracking in living tissue. PloS one 5, e11639.

Snaidero, N., Mobius, W., Czopka, T., Hekking, L. H., Mathisen, C., Verkleij, D., Goebbels, S., Edgar, J., Merkler, D., Lyons, D. A., Nave, K. A., Simons, M., 2014. Myelin membrane wrapping of CNS axons by PI(3,4,5)P3-dependent polarized growth at the inner tongue. Cell 156, 277-290. 
Sobottka, B., Harrer, M. D., Ziegler, U., Fischer, K., Wiendl, H., Hunig, T., Becher, B., Goebels, N., 2009. Collateral bystander damage by myelin-directed CD8+ T cells causes axonal loss. Am J Pathol 175, 1160-1166.

Sobottka, B., Ziegler, U., Kaech, A., Becher, B., Goebels, N., 2011. CNS live imaging reveals a new mechanism of myelination: the liquid croissant model. Glia 59, 1841-1849.

Sospedra, M., Martin, R., 2005. Immunology of multiple sclerosis. Annu Rev Immunol 23, 683-747.

Stevens, B., Porta, S., Haak, L. L., Gallo, V., Fields, R. D., 2002. Adenosine: a neuronglial transmitter promoting myelination in the CNS in response to action potentials. Neuron 36, 855-868.

Wake, H., Lee, P. R., Fields, R. D., 2011. Control of local protein synthesis and initial events in myelination by action potentials. Science 333, 1647-1651.

Watkins, T. A., Emery, B., Mulinyawe, S., Barres, B. A., 2008. Distinct stages of myelination regulated by gamma-secretase and astrocytes in a rapidly myelinating CNS coculture system. Neuron 60, 555-569.

Werner, C., Engelhard, K., 2007. Pathophysiology of traumatic brain injury. Br J Anaesth 99, 4-9.

Xiao, L., Hu, C., Yang, W., Guo, D., Li, C., Shen, W., Liu, X., Aijun, H., Dan, W., He, C., 2013. NMDA receptor couples Rac1-GEF Tiam1 to direct oligodendrocyte precursor cell migration. Glia 61, 2078-2099.

Zonta, B., Tait, S., Melrose, S., Anderson, H., Harroch, S., Higginson, J., Sherman, D. L., Brophy, P. J., 2008. Glial and neuronal isoforms of Neurofascin have distinct roles in the assembly of nodes of Ranvier in the central nervous system. J Cell Biol 181, 1169-1177. 


\section{Figure legends}

Figure 1. Live visualisation of OL-axon interactions and myelination. A. Ex vivo images indicating initial OL-axon interactions in the spinal cord. OL generated from cGFP expressing neurospheres were transferred into shiverer Thyl-CFP mice, before imaging in acute spinal cord explants prepared at 15 days post transplantation. Ai-iii. Time-lapse images indicate the initial stages of myelination as a $\mathrm{GFP}^{+} \mathrm{OL}$ process (green) contacts (Ai) and then extends (Aii-iii) along a CFP+ axon. (from Ioannidou et al., 2012). B. Newly differentiated OL exhibit a brief period of myelin segment formation. Bi. In vivo time-lapse images from nkx2.2a:mGFP zebra fish depicting the sequential growth of nascent myelin segments (cyan). In this example the first and last segments to be formed are identified as \#1 and \#15 respectively. Scale $=10 \mu \mathrm{m}$. Bii. Quantification of the number of new myelin segments added over time. Time zero is defined as the frame at which the first segment appears. Cyan line indicates the cell depicted in $\mathrm{Bi}$. (reproduced with permission from Czopka et al., 2013). C. Ex vivo imaging of demyelination and remyelination in cerebellar slice cultures. Confocal imaging of PLPeGFP signals from injury (Ci) and control (Cii) treated slices obtained before ( $0 \mathrm{~h})$, during (43 h), and after $(53-77 \mathrm{~h})$ treatment with MOG antibody and complement. Note remyelination is first observed in regions containing persistent $\mathrm{GFP}^{+} \mathrm{OL}$. Scale $=50 \mu \mathrm{m}$. (reproduced with permission from Harrer et al., 2009).

Figure 2. Single-molecule imaging of MBP diffusion properties within myelin sheets. A. Single molecule diffusion tracking and FRAP within primary mice oligodendrocytes expressing a chimeric molecule containing the photoswitchable fluorophore Dendra2, connected to an integral membrane protein (Dendra2-TM, Ai), or both Dendra2 and MBP (Dendra2-TM-MBP, Aii). Activation of Dendra2 with UV light in a $5 \mu \mathrm{m}$ by $5 \mu \mathrm{m}$ region of the sample enabled time-lapse imaging of photoconverted Dendra2 (red). Chimeric 
protein lacking lacking MBP (Ai) undergoes diffusion over time. In contrast diffusion is not apparent when MBP is attached to the chimeric protein (Aii ) suggesting that MBP has an anchoring effect on the proteins. Scale bar $10 \mu \mathrm{m}$. B. Decay of the photoconverted fluorescent signal over the course of the experiment. Decay is greater in chimeric protein lacking MBP (black) compared to the fusion containing MBP (grey). C. Average decay of Dendra2 signal over the time-course of the experiment is significantly greater in chimeric protein lacking MBP indicating that MBP reduces the diffusion of Dendra2 labeled molecules. D\&E. Fluorescence recovery of GFP signal following photobleaching. Cells were transfected with similar constructs to those described in A-C, but with GFP replacing Dendra2, and GFP was photobleached within a $5 \mu \mathrm{m}$ by $5 \mu \mathrm{m}$ region of the sample. D. Recovery of GFP signal is stronger in chimeric protein lacking MBP (black) compared to the fusion protein containing MBP (grey). E. Average recovery of GFP signal is significantly reduced when MBP is attached to the chimeric protein indicating lower diffusion of GFP labelled proteins. ${ }^{*}$ significance at $\mathrm{P}<0.01$, *** significance at $\mathrm{P}<0.001$. Histograms show Mean \pm SE. (From Aggarwal et al., 2013).

Figure 3. Schematic illustrating the main model systems used for live-imaging of CNS OL-axon dynamics and myelination. Important features of the models are presented along with key findings on myelin formation and injury that each model has supported. Ensemble and Single Molecule Imaging methodologies are also compared to highlight particular advantages and disadvantages of these approaches, and key advances they have enabled in our understanding of myelin biology. 

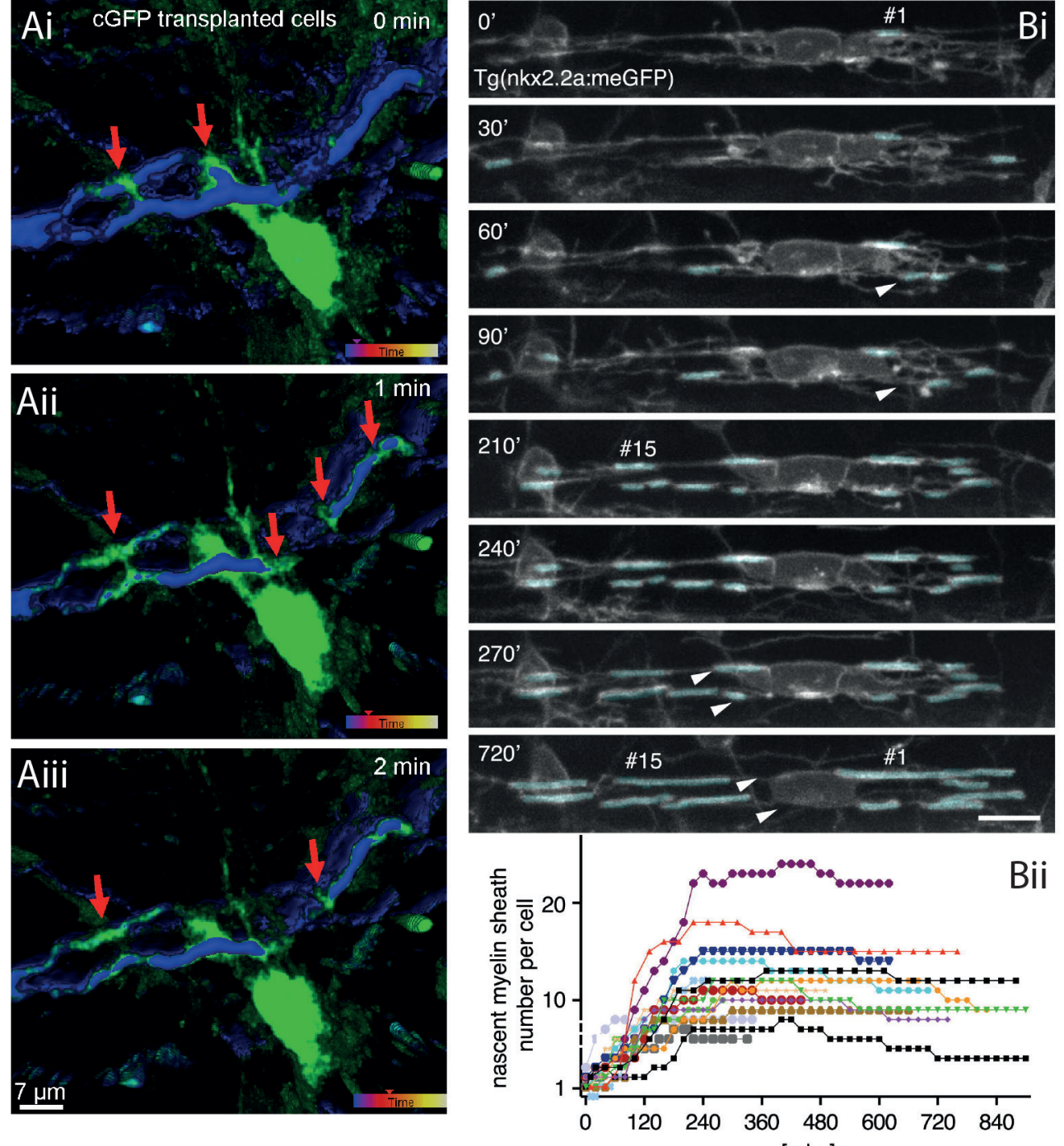

Bii

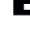

$$
\begin{array}{llllllll}
0 & 120 & 240 & 360 & 480 & 600 & 720 & 840
\end{array}
$$

c
$0 \mathrm{~h}$

$43 \mathrm{~h}$
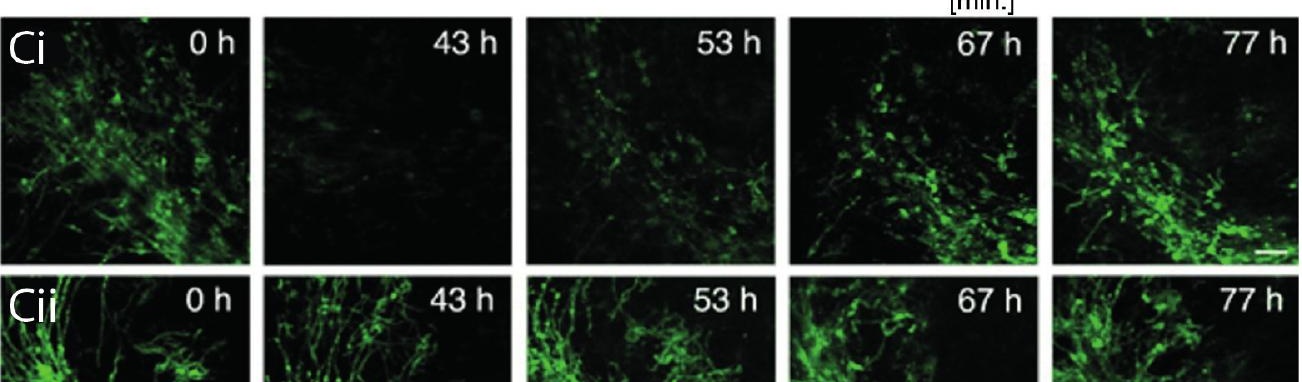

2528
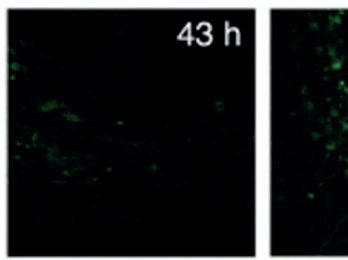

$43 \mathrm{~h}$
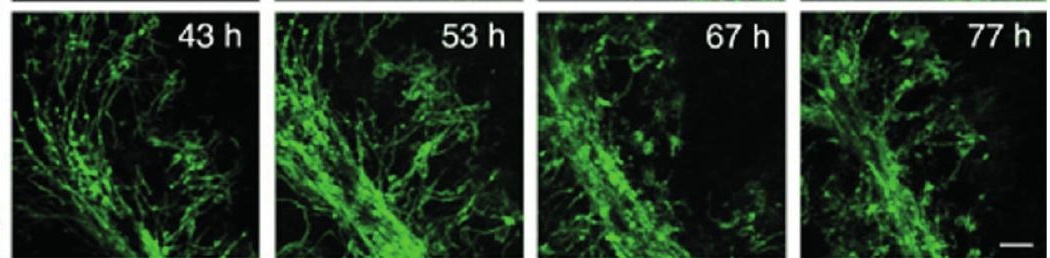


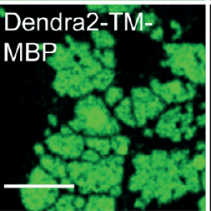

B

- Dendra2-TM

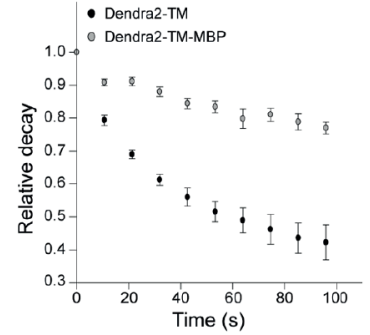

C

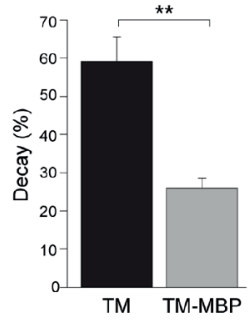

D

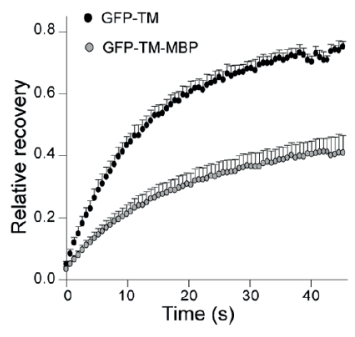

E

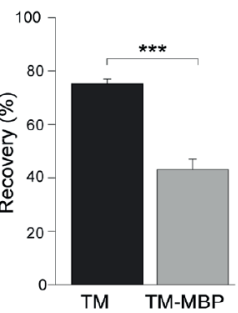




\section{Model System}

\section{In Vitro}

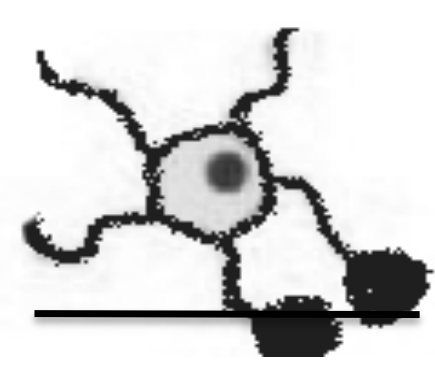

\section{$\square$ Wide usage}

provides critical mass of knowledge

$\square$ Precise timing of OPC seeding and differentiation $\square$ FP labeled OPC provide flexible low cost alternative to transgenic mice

$凶$ Lack most of the cell types and cell-cell interactions present in the CNS

1. Brief time-window for initiation of new myelin segments (Watkins et al. 2008)

2. Myelination begins with a spiral wrapping of axons by OPC processes (Ioannidou et al. 2012)

\section{Ex Vivo}

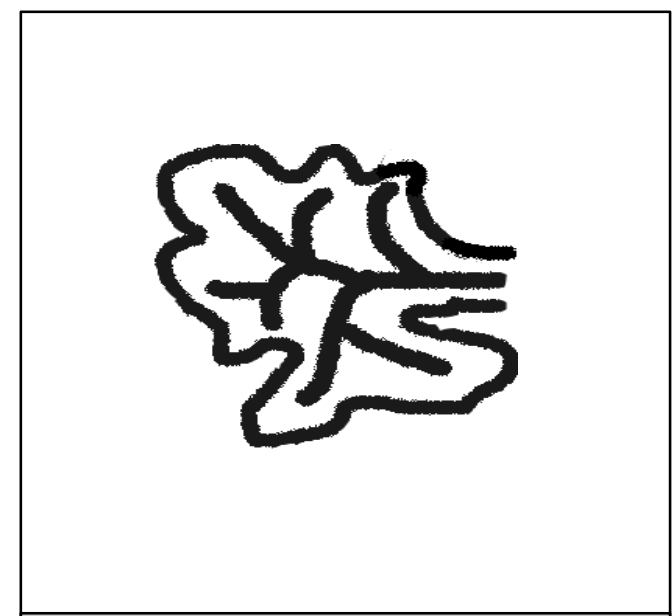

$\square$ CNS cytoarchitecture And interactions largely preserved

$\square$ Convenient for longterm imaging of OPC migration, development and myelination

$\square$ Established models of myelin injury available

\section{冈 Slice cultures from} mature CNS difficult to establish

3. Myelin initiated at multiple axonal loci before growing via lateral spreading (loannidou et al. 2012)

4. OL surviving immune mediated injury contribute to myelin repair (Harrer et al. 2009)

\section{In Vivo}

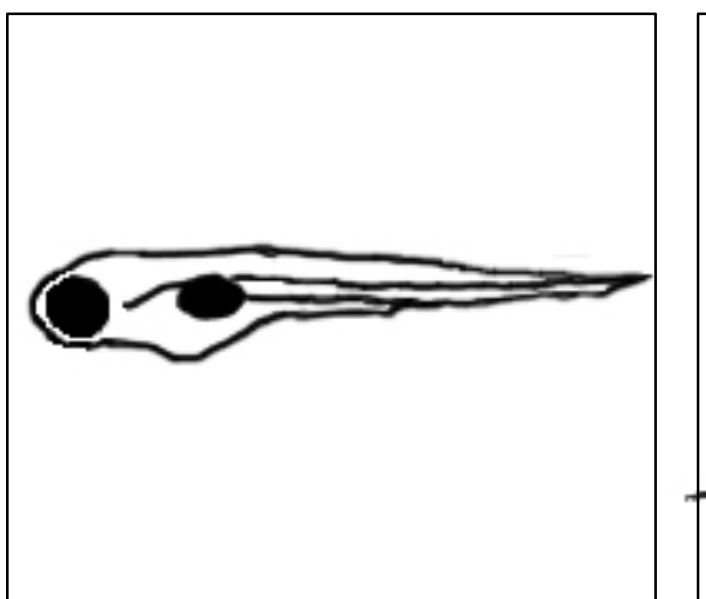

$\square$ Rapid generation of transgenic lines

$\square$ Convenient long-term imaging of the CNS in the intact animal

$\square$ Low cost of animal Husbandry

冈 Not suited to imaging adult myelin injury

囚 Lack of adaptive Immune system limits scope of myelin disease modeling

\section{OL spacing} determined by OPC self-avoidance via process mediated surveillance (Kirby et al. 2006)

6. Activity regulates myelin sheath number and maintenance (Mensch et al. 2015; Hines et al., 2015)

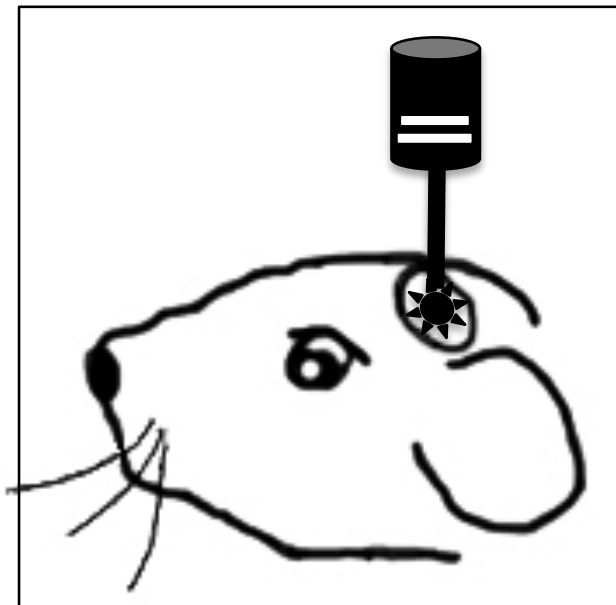

$\square$ Long-term imaging of $\mathrm{OL}$ and myelin in a native mammalian CNS

$\square$ Availability of clinically relevant myelin injury models

冈 Time-consuming and costly surgeries required to provide optical access

冈 Imaging of deep white matter limited by current technologies

\section{Adult OPC}

respond to injury by

migrating into spaces vacated by dying OPC

(Hughes et al. 2013)

\section{Ensemble}

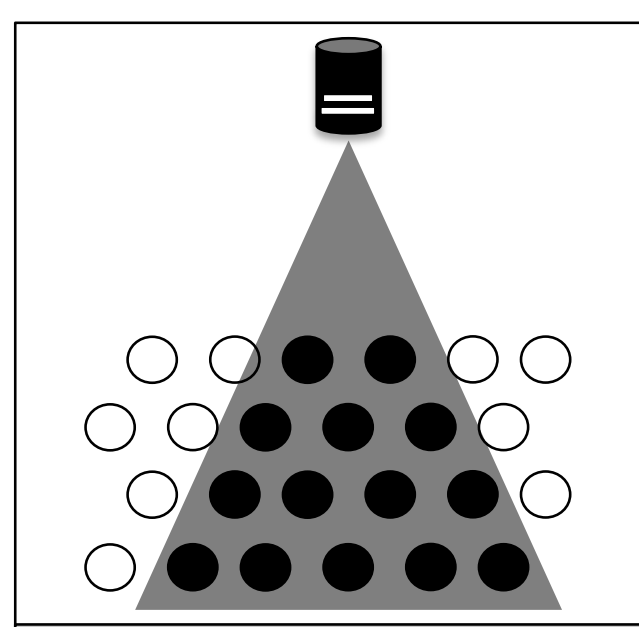

$\square$ Monitor larger features, e.g. myelin internodes or OL processes

$\square$ Readily implemented in in vitro, ex vivo and in vivo models

凶 Unable to resolve the behaviour of individual elements within a population of fluorescently labeled molecules

\section{See items 1-7.}

\section{SMI}

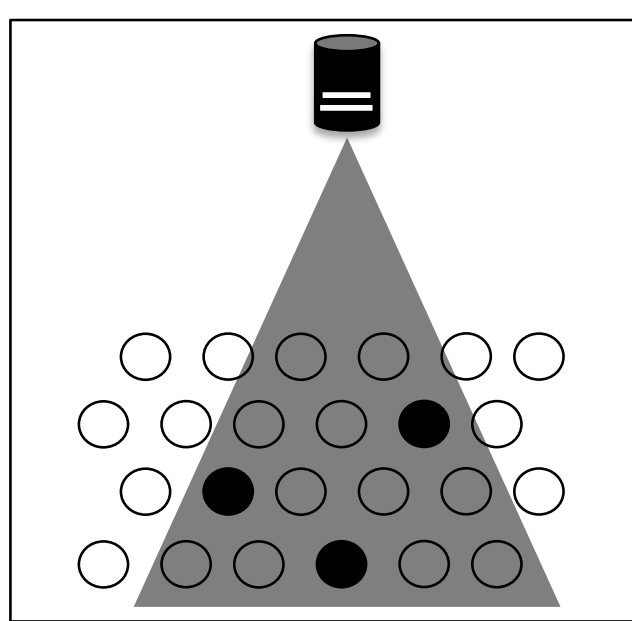

$\square$ Track behaviour of sub-population of fluorophores in space or time

\section{$\square$ Potential to} determine quantitative data on disease-related molecular degeneration, and drug kinetics

冈 Limited to in vitro CNS models by current technologies

\section{- MBP mRNA}

transported to developing myelin (Ainger et al. 1993)

Self assembly of MBP molecules in the inner leaflet of myelin (Aggarwal et al. 2013) 